Article

\title{
Enhanced Oral Bioavailability, Anti-Tumor Activity and Hepatoprotective Effect of 6-Shogaol Loaded in a Type of Novel Micelles of Polyethylene Glycol and Linoleic Acid Conjugate
}

\author{
Huiyun Zhang ${ }^{+}\left(\mathbb{D}\right.$, Qilong Wang ${ }^{\dagger}$, Congyong Sun ${ }^{\dagger}$, Yuan Zhu, Qiuxuan Yang, Qiuyu Wei, \\ Jiaxin Chen, Wenwen Deng, Michael Adu-Frimpong, Jiangnan $\mathbf{Y u}^{*}$ and Ximing $\mathrm{Xu}$ * \\ Center for Nano Drug/Gene Delivery and Tissue Engineering, Department of Pharmaceutics, \\ School of Pharmacy, Jiangsu University, Zhenjiang 212013, China; zhanghuiyun1111@163.com (H.Z.); \\ wq1001wq1001@163.com (Q.W.); horizonpacino@sina.cn (C.S.); zhuyuanemail@126.com (Y.Z.); \\ yangqiuxuan1016@126.com (Q.Y.); weiqiuyu0922@163.com (Q.W.); 18352864633@163.com (J.C.); \\ deng4956@yeah.net (W.D.); 5103160103@stmail.ujs.edu.cn (M.A.-F.) \\ * Correspondence: yjn@ujs.edu.cn (J.Y.); xmxu@ujs.edu.cn (X.X.); Tel.: +86-5118-503-845 (J.Y. \& X.X.); \\ Fax: +86-5118-503-8451 (J.Y. \& X.X.) \\ + These authors contributed equally to this work.
}

Received: 19 December 2018; Accepted: 1 March 2019; Published: 6 March 2019

\begin{abstract}
However, the treatment effectiveness of 6-shogaol is limited by poor water solubility, poor oral absorption and rapid metabolism. Herein, 6-shogaol loaded in micelles (SMs) were designed to improve 6-shogaol's solubility and bioavailability. The micelles of a PEG derivative of linoleic acid ( $\mathrm{mPEG}_{2 \mathrm{k}}-\mathrm{LA}$ ) were prepared by the nanoprecipitation method with a particle size of $76.8 \mathrm{~nm}$, and entrapment of $81.6 \%$. Intriguingly, SMs showed a slower release in phosphate buffer saline (PBS) $(\mathrm{pH}=7.4)$ compared to free 6-shogaol while its oral bioavailability increased by 3.2-fold in vivo. More importantly, the in vitro cytotoxic effect in HepG2 cells of SMs was significantly higher than free 6-shogaol. Furthermore, SMs could significantly improve the tissue distribution of 6-shogaol, especially liver and brain. Finally, SMs showed a better hepatoprotective effect against carbon tetrachloride (CCl4)-induced hepatic injury in vivo than free 6-shogaol. These results suggest that the novel micelles could potentiate the activities of 6-shogaol in cancer treatment and hepatoprotection.
\end{abstract}

Keywords: 6-shogaol; cytotoxic effect; HepG2 cells; micelles; hepatoprotection

\section{Introduction}

Ginger, the dried rhizome of Zingiber officinale, is not only a popular spice and seasoning, but also a traditional Chinese medicine used to treat many diseases such as nausea, diarrhea and cold [1]. Recently, gingerols, the main chemical components of ginger, have been found responsible for pharmacological effects including analgesic [2], antipyretic [3], cardiotonic [4], hypothermia inducing [5], and cancer prevention [6]. 6-shogaol is one of the shogaols obtained from Gingerols via dehydration during processing and storage. 6-shogaol exhibits significant antioxidant, anti-proliferative and anti-inflammation activity [7]. 6-shogaol is reported to induce cell cycle arrest coupled with autophagy and apoptosis in various cancer cells including hepatocellular carcinoma (HCC) cell, human non-small cell lung cancer A549 cells and human colon adenocarcinoma (HT-29) cells [8-10]. 6-shogaol has also been previously reported to possess anti-inflammatory effects by downregulating nitric oxide synthase and COX-2 gene expression [11], while reducing the levels of $\beta$-glucuronidase and lactate dehydrogenase [12]. 
The potentiality of 6-shogaol is supported by several lines of evidence as an agent for the prevention and treatment of a variety of cancers including gastrointestinal, melanoma, breast, lung, head and neck [13-17]. Also, various in vitro and in vivo studies showed that 6-shogaol demonstrates more potent anti-inflammatory activity than 6-gingerol and another widely studied phytochemical, curcumin [18]. However, low bioavailability alongside poor solubility of 6-shogaol hinder its clinical application, probably due to poor absorption, hydrophobicity, extreme instability, rapid metabolism, with concomitant elimination [19,20]. Recently, nanotechnology (polymer nanoparticles or micelles, liposomes, inorganic nanoparticles and nano-emulsions) have shown a huge advantage in enhancing the solubility of lipophilic compounds, oral absorption, bioavailability as well as reducing medicinal herb doses and toxicity, thereby improving the target ability and therapeutic effect compared with traditional Chinese herbal preparations [21,22]. Among them, micelles have already received a lot of attention due to their unique peculiarities like a hydrophilic outer shell coupled with a hydrophobic inner core. These qualities impart on micelles' water dissolvability, which result in the spontaneous production of micelles with the capability of encapsulating and solubilizing poorly aqueous-soluble drugs $[23,24]$. Comparatively, self-assembled micelles could provide several advantages to drug delivery systems because of their high drug loading capacity, low dose of formulation required and long circulation time $[25,26]$.

Currently, several PEGylated small amphiphilic molecules such as D- $\alpha$-tocopheryl polyethylene glycol succinate (TPGS), amphiphilic PEG1000-DOX conjugate and PEG-all trans-retinoic acid conjugates are being used as materials for the fabrication of micelles [27-29]. Compared with clinical formulation, docetaxel-loaded TPGS2k micelles showed a lower critical micelle concentration (CMC) and achieved a sustained and controlled drug delivery. Furthermore, docetaxel-loaded TPGS2k micelles enhanced the cytotoxicity on cancer cell lines with the side effects of docetaxel being modulated [30]. As a P-glycoprotein (P-gp) inhibitor, TPGS micelles for oral delivery of paclitaxel have been demonstrated to have superior performance in vivo as evidenced by their enhanced absorption and biological availability [31]. Encapsulating 6-shogaol in micelles will therefore gain benefits from the aforementioned advantages that are associated with such a micelle delivery system.

In the present study, PEG derivative of linoleic acid $\left(\mathrm{mPEG}_{2 \mathrm{~K}}-\mathrm{LA}\right)$ was firstly employed as a material for forming micelles to encapsulate 6-shogaol and enhance its solubility. The formulated 6-shogaol loaded micelles (SMs) significantly slowed the drug release in stimulated media of gastro-intestinal tract and increased the sensitivity of tumor cells to the prototype drug. The oral bioavailability, tissue biodistribution and hepatoprotective activity were investigated to evaluate the effectiveness of SMs in delivering 6-shogaol, as well as its bioactivity in vivo. Collectively, this study could provide an experimental basis for the further development and application of self-assembly nano drug delivery systems for enhancing the bioactivity of hydrophobic drugs.

\section{Materials and Methods}

\subsection{Materials}

6-shogaol (98\% purity, analytical-grade reagent) was purchased from Aladdin Industrial Corporation (Shanghai, China). Curcumin was obtained from J\&K Scientific Co. Ltd. (Beijing, China). Ginger extractive (10\% gingerol) was purchased from Nanjing Zelang Biological Technology Co. Ltd. (Nanjing, China). $\mathrm{mPEG}_{2 \mathrm{~K}}$-LA was synthesized in our laboratory [32]. (3-(4,5-Dimethylthiazol-2-yl)2,5-diphenyltetrazolium bromide (MTT) and trypsin were provided by the Beyotime Institute of Biotechnology (Jiangsu, China). Diagnostic kits for assaying serum aspartate aminotransferase (AST), alanine aminotransferase (ALT) and the glutathione peroxidase (GSH-Px), total superoxide dismutase (T-SOD) and the levels of malondialdehyde (MDA) were purchased from Nanjing Jian Cheng Bioengineering institute (Nanjing, China). Chromatographically pure methanol and acetonitrile were procured from Sinopharm Chemical Reagent Technology Co. Ltd. (Shanghai, China). 


\subsection{Preparation of 6-Shogaol from Ginger Extractive}

Concisely, the ginger extract (150 g) was first extracted with ethyl acetate, and the organic solvent layer was concentrated up to dryness under a rotary evaporator. Then, a silica gel column was used to separate the 6-shogaol from ethyl acetate extract with gradient elution consisting of petroleum ether and ethyl acetate (5:1, 4:1, 3:1, 2:1 and 5:3) with $500 \mathrm{~mL}$ of each mixture. The crude samples were purified further using a $\mathrm{C} 18$ silica gel column and eluted with different proportions of methanol and water with ratios ranging from 50/50 to 100/0. The obtained purified samples were identified with ESI-MS (Figure S1) and ${ }^{1} \mathrm{H}-\mathrm{NMR}$ (Figure S2). According to the calibration curve of HPLC, the obtained 6-shogaol had a purity of $96.67 \%$. The chemical structure was provided in Figure S3. Chemical formula: $\mathrm{C}_{17} \mathrm{H}_{24} \mathrm{O}_{3}$. ESI-MS (m/z): $299.16[\mathrm{M}+\mathrm{Na}]^{+} .{ }^{1} \mathrm{H}-\mathrm{NMR}\left(400 \mathrm{MHz}, \mathrm{CDCl}_{3}, \mathrm{ppm}\right): \delta 0.91\left(\mathrm{t}, 3 \mathrm{H},-\mathrm{CH}_{3}\right)$, $1.25-1.38\left(\mathrm{~m}, 4 \mathrm{H},-\mathrm{CH}_{2}\right), 1.47\left(\mathrm{dt}, 2 \mathrm{H}, J=7.3\right.$ and $\left.14.6 \mathrm{~Hz},-\mathrm{CH}_{2}-\right), 2.21(2 \mathrm{H}, \mathrm{dd}, J=6.3$ and $14.0 \mathrm{~Hz})$, $2.87\left(4 \mathrm{H}, \mathrm{tt}, J=5.9\right.$ and $12.0 \mathrm{~Hz},-\mathrm{CH}_{2}-$ between $\mathrm{Ph}$ ring and ketone), $3.88(\mathrm{~s}, 3 \mathrm{H},-\mathrm{OMe}), 6.11(1 \mathrm{H}, \mathrm{dt}$, $J=1.5$ and $15.9 \mathrm{~Hz},=\mathrm{CH}-), 6.68(1 \mathrm{H}, \mathrm{dd}, J=2.0$ and $8.0 \mathrm{~Hz},=\mathrm{CH}-), 6.72(1 \mathrm{H}, \mathrm{d}, J=1.8 \mathrm{~Hz}, \mathrm{ArH}), 6.84$ $(2 \mathrm{H}, \mathrm{m}, \mathrm{ArH})$.

\subsection{Solubility of 6-Shogaol}

The solubility of 6-shogaol was determined according to our earlier reported method [22]. Simply, $20 \mathrm{mg}$ of 6-shogaol $(20 \mathrm{mg}$ ) was added to $1 \mathrm{~mL}$ of a different dissolution medium, followed by incubation in a water bath shaker for $100 \mathrm{rpm}$ at $37^{\circ} \mathrm{C}$ for 3 days. The suspension was then centrifuged at 10,000 rpm for $20 \mathrm{~min}$ to remove the insoluble 6-shogaol. Then, the concentration of supernatant was measured with established HPLC method.

\subsection{Preparation of 6-Shogaol Loaded Micelles (SMs)}

Self-assembled micelles were prepared via the nanoprecipitation method as previously reported with slight modifications [33]. Briefly, 6-shogaol $(10 \mathrm{mg})$ and $\mathrm{mPEG}_{2 \mathrm{~K}}-\mathrm{LA}(100 \mathrm{mg})$ were completely dissolved in $200 \mu \mathrm{L}$ ethanol solution $(200 \mu \mathrm{L})$. The ethanol solution was added drop-wise to $2 \mathrm{~mL}$ water $(2 \mathrm{~mL})$ at room temperature, alongside mechanical stirring ( 600-800 revolutions per minute $(\mathrm{rpm}))$ while self-assembly of NMs occurred spontaneously. The organic solvent-free SMs were obtained after evaporating the ethanol in the nano-formulation. The SMs were filtered through a $0.22 \mu \mathrm{m}$ filter membrane, and then lyophilized prior to storage at $4{ }^{\circ} \mathrm{C}$.

\subsection{HPLC Analysis Method for Measuring 6-Shogaol Concentration}

6-shogaol levels in micelle, plasma and tissue samples were measured via an RP-HPLC method. HPLC analysis was carried out using a Shimadzu Scientific instrument equipped with an LC-20AT pump and an SPD-20A UV-Vis detector (Shimadzu, Kyoto, Japan) on a Symmetric C18 column $\left(4.6 \mathrm{~mm} \times 150 \mathrm{~mm}, 5 \mu \mathrm{m}\right.$, Waters, Milford, MA, USA) with column temperature of $30^{\circ} \mathrm{C}$. The flow rate was set at $1.0 \mathrm{~mL} / \mathrm{min}$ while the detection wavelength was $230 \mathrm{~nm}$. A $70 \%$ methanol-water was chosen as the mobile phase for measuring encapsulation efficiency (EE) and in vitro release, while $65 \%$ methanol-water was used for the analysis of bioavailability and tissue biodistribution studies. The system suitability test results of the formulation, PK and tissue analysis methods were depicted in Figures S4-S6. The linear regression equation of 6-shogaol in vitro analysis was $\mathrm{Y}=81883 \mathrm{X}-$ $44299\left(\mathrm{n}=3, R^{2}=0.9997\right)$, where $\mathrm{Y}$ represented the peak area of samples and $\mathrm{X}$ represented the sample concentrations of 6-shogaol. The typical equation of the standard curves of the peak area ratio to the concentration, the linear range and regression coefficients of 6-shogaol in plasma and tissue homogenates were summarized in Table S1. The precision (intra-day and inter-day precision) and accuracy (relative recovery) of the analysis method were assessed at 3 levels of QC samples (in vivo) in different matrices $(0.35 \mu \mathrm{g} / \mathrm{mL}, 1.5 \mu \mathrm{g} / \mathrm{mL}$ and $7.5 \mu \mathrm{g} / \mathrm{mL}$ in rat plasma; $0.15 \mu \mathrm{g} / \mathrm{mL}, 0.375 \mu \mathrm{g} / \mathrm{mL}$ and $0.75 \mu \mathrm{g} / \mathrm{mL}$ in heart, spleen, lung, brain, liver, kidney, stomach and intestine), and the results were shown in Table S2. The extraction recovery of 6-shogaol in plasma, heart, liver, spleen, lung, kidney and 
brain were within the range of $76.23-87.12 \%, 73.43-87.73 \%, 72.51-82.78 \%, 74.78-87.18 \%, 76.34-87.50 \%$, $79.11-85.45 \%, 74.86-86.47 \%, 82.41-84.21 \%, 72.46-84.69 \%$ (data shown in Table S2), respectively.

\subsection{Characterization of SMs}

The hydrodynamic diameters and Zeta potential (ZP) of SMs were carried out using a Zeta Potential/Particle Size analyzer (Brookhaven Instruments Corporation, Holtsville, NY, USA). The morphology and particle size of the SMs were measured using transmission electron microscopy (TEM) (JEM-2100, JEOL, Tokyo, Japan). The diluted SMs samples $(1 \mathrm{mg} / \mathrm{mL})$ were dropped onto a copper grid and allowed to stand for $3 \mathrm{~min}$. The sample was dried with filter paper alongside staining with phosphotungstic acid solution $(2 \%, \mathrm{w} / \mathrm{v})$ for 1-2 min followed by air-drying. Subsequently, the prepared samples were viewed under TEM operated at an accelerating voltage of $200 \mathrm{kV}$.

\subsection{6-Shogaol Loading and Encapsulation Efficiency}

Drug loading (DL) and encapsulation efficiency (EE) were measured as follows. Freeze-dried 6-shogaol micelle $(1 \mathrm{mg})$ was dissolved in $5 \mathrm{~mL}$ of acetonitrile. After ultrasonication for $5 \mathrm{~min}$, the solution was filtered through a $0.45-\mu \mathrm{m}$ filter membrane. The drug concentration in the filtrate was quantified using HPLC. The EE and DL of micelles were calculated according to Equations (1) and (2), respectively.

$$
\begin{gathered}
\text { EE }(\%)=\text { Experimental drug loading/Theoretical drug loading } \times 100 \% \\
\text { DL }(\%)=\text { Weight of drug/Weight of carrier and drug } \times 100 \%
\end{gathered}
$$

\subsection{In Vitro Release of SMs}

In vitro release experiments of 6-shogaol stock solution and SMs were measured via the dialysis method under sink conditions. Briefly, an aliquot $(1.0 \mathrm{~mL}, 0.5 \mathrm{mg} / \mathrm{mL})$ of 6 -shogaol stock solution and SMs were transferred into dialysis bags $(\mathrm{MW}=3000)$ and dialyzed against $80 \mathrm{~mL}$ of phosphate buffer solutions of different $\mathrm{pH}$ values (PBS, 1.2 and 7.4, respectively) at $37^{\circ} \mathrm{C}$ at a constant speed of $70 \mathrm{rpm}$. Aliquots $(1 \mathrm{~mL})$ were taken from dialysate at predetermined times $(0 \mathrm{~h}, 0.5 \mathrm{~h}, 1 \mathrm{~h}, 2 \mathrm{~h}, 4 \mathrm{~h}, 6 \mathrm{~h}, 8 \mathrm{~h}, 12 \mathrm{~h}$ and $24 \mathrm{~h}$ ), and subsequently replaced with an equal volume of preheated fresh release media. The 6-shogaol concentration of collected samples filtered through a $0.22-\mu \mathrm{m}$ cellulose nitrate membrane was analyzed with HPLC. The cumulative release (\%) was calculated by the weight ratio of released 6-shogaol to total 6-shogaol.

\subsection{Cell Viability Assay}

HepG2 cells were seeded in 96-well plates at a density of 4,000 cells/well. Cells were incubated for $24 \mathrm{~h}$ at $37^{\circ} \mathrm{C}$ under fully humidified conditions containing $5 \% \mathrm{CO}_{2}$. The cells were then treated with a series of concentrations $(5 \mu \mathrm{M}, 10 \mu \mathrm{M}, 20 \mu \mathrm{M}, 50 \mu \mathrm{M}, 75 \mu \mathrm{M}, 100 \mu \mathrm{M}$, and $200 \mu \mathrm{M})$ of samples (free 6-shogaol and SMs) in $100 \mu \mathrm{L}$ culture medium. After incubation for $72 \mathrm{~h}, 20 \mu \mathrm{L}$ of MTT solution $\left(5 \mathrm{mg} / \mathrm{mL}\right.$ ) was added into each well, and the plate was incubated further at $37^{\circ} \mathrm{C}$ for $4 \mathrm{~h}$. After the addition of DMSO $(100 \mu \mathrm{L})$ to solubilize the formazan crystals, the optical density was determined using a microplate plate reader (BioTek, Winooski, Vermont, USA) plate reader at $595 \mathrm{~nm}$. The cell viability rate $(\mathrm{VR})$ was calculated using the following equation: $\operatorname{VR}(\%)=\left(\mathrm{A}^{\mathrm{s}}-\mathrm{A}^{0}\right) /\left(\mathrm{A}^{1}-\mathrm{A}^{0}\right) \times 100 \%$, where $A^{s}, A^{0}$ and $A^{1}$ are the absorbance of sample group, negative and positive control group [34].

\subsection{In Vivo Sample Treatment}

Tissue homogenate samples (liver- $0.3 \mathrm{~g}$ ) were prepared after the addition of normal saline $(2 \mathrm{~mL})$ and homogenate for $2 \mathrm{~min}$. Afterward, $50 \mu \mathrm{L}$ of internal standard solution (curcumin, $5 \mu \mathrm{g} / \mathrm{mL}$ ) was added to $0.5 \mathrm{~mL}$ of each of the homogenates or $200 \mu \mathrm{L}$ of plasma. After mixing completely, the samples were extracted with $3 \mathrm{~mL}$ ethyl acetate. The upper organic layer was taken and dried with nitrogen at 
$40{ }^{\circ} \mathrm{C}$ after centrifugation at $4000 \mathrm{rpm}$ for $10 \mathrm{~min}$. The samples were later reconstituted in $0.1 \mathrm{~mL}$ of $65 \%$ methanol as mobile phase. The supernatant was analyzed using HPLC after centrifugation at $10,000 \mathrm{rpm}$ for $10 \mathrm{~min}$.

\subsection{Oral Pharmacokinetic Study of Micelles}

Twelve male Sprague-Dawley (SD) rats (male, 5-7 weeks, $220 \pm 20 \mathrm{~g}$ ) were obtained from Laboratory for Animals Care and Use of Jiangsu University, Jiangsu (Zhenjiang, Jiangsu Province, China, SCXK (SU) 20130036). This study was approved by the Experimental Animal Care and Use Committee of the Science and Technology, Zhenjiang, China. Animal are complied with institutional guidelines and regulations (JUC-201727462). All the rats were maintained in an environmentally controlled room $\left(23^{\circ} \mathrm{C}, 12 \mathrm{~h}\right.$ dark light cycle) with free access to standard laboratory food and water for 7 days before the experiments. Before oral administration, the entire rats were randomly divided into two groups with 6 cases each, which were then fasted for $12 \mathrm{~h}$ but given only water. The two groups were orally administrated with free 6-shogaol suspension $[5 \mathrm{mg} / \mathrm{mL}$, in $0.5 \%(\mathrm{v} / \mathrm{v})$ castor oil mixture, $100 \mathrm{mg} / \mathrm{kg}$, and SMs of equivalent 6-shogaol dose $(5 \mathrm{mg} / \mathrm{mL}$, in normal saline, $100 \mathrm{mg} / \mathrm{kg}$ ). Blood samples $(600 \mu \mathrm{L}$ each) were withdrawn at appointed time periods $(0.25,0.5,0.75,1,1.5,2,4,6$, 8 and $12 \mathrm{~h}$ ). Plasma ( $200 \mu \mathrm{L}$ each) was obtained from blood samples after centrifuging at $3000 \mathrm{rpm}$ and then were pretreated as described under the "Sample pretreatment" section. The 6-shogaol content in supernatant was determined using HPLC analysis. The pharmacokinetic parameters of peak concentration $\left(\mathrm{C}_{\max }\right)$, time of peak concentration $\left(\mathrm{T}_{\max }\right)$, area under the plasma concentration-time curve $\left(\mathrm{AUC}_{0-12 \mathrm{~h}}\right)$ and elimination half-life $\left(\mathrm{t}_{1 / 2}\right)$ were determined using BAPP 2.3 pharmacokinetic software supplied by the Center for Drug Metabolism of the China Pharmaceutical University.

\subsection{Tissue Distribution of SMs}

Thirty Kunming mice (male, 4-6 weeks, weighing 18 g-22 g) were obtained from Laboratory for Animals Care and Use of Jiangsu University, Jiangsu (Zhenjiang, Jiangsu Province, China, SCXK (SU) 20130036). Prior to oral administration, all of the mice were randomly and equally divided into 2 groups, and then fasted for $12 \mathrm{~h}$ and fed with only water. The first group was orally administered with free 6-shogaol suspension [ $5 \mathrm{mg} / \mathrm{mL}$ in $0.5 \%(\mathrm{v} / \mathrm{v})$ castor oil mixture] at a dose of $100 \mathrm{mg} / \mathrm{kg}$, while the other group was treated with the same content of 6-shogaol in the SMs. At specific times $(0.5 \mathrm{~h}, 1 \mathrm{~h}$ and $4 \mathrm{~h})$ after the oral administration, the blood was taken from the mice's eye socket and the tissues viz. heart, spleen, lung, liver, kidney, stomach, small intestine and brain were collected from the sacrificed mice. All tissue samples were rinsed with ice-cold saline and blotted dry. The samples were then weighed and frozen at $-20{ }^{\circ} \mathrm{C}$ for further analysis. The drug content of tissue was measured via HPLC analysis. The ratio of the content of tissue and blood was calculated as the relative targeting drug delivery index (DDI).

\subsection{Determination of Hepatoprotective Effect in Vivo}

The hepatoprotective effect in vivo was tested in mice using $\mathrm{CCl}_{4}$-induced hepatotoxicity model. Kunming mice were randomly divided into five groups, each of ten cases. Mice of the $1^{\text {st }}$ (normal control) and $2^{\text {nd }}$ (intoxicated control) groups were treated with a single dose of gastric gavage physiological saline $\left(20 \mathrm{~mL} / \mathrm{kg}\right.$ body weight) via oral administration. Animals of the $3^{\text {rd }}, 4^{\text {th }}$ and $5^{\text {th }}$ groups were respectively given sylimarin ( $100 \mathrm{mg} / \mathrm{kg}$ body weight per day), free 6-shogaol $(100 \mathrm{mg} / \mathrm{kg}$ ) and SMs $(100 \mathrm{mg} / \mathrm{kg})$ orally each day for one week. At $6 \mathrm{~h}$ after last dose, the hepatotoxicity model was induced in all mice except those in the normal group by oral administration of $\mathrm{CCl}_{4}$ at a single dose of $\mathrm{CCl}_{4}$ and castor oil mixture $\left(0.3 \% \mathrm{v} / \mathrm{v}, 5 \mathrm{~mL} / \mathrm{kg}\right.$ body weight). At $24 \mathrm{~h}$ after $\mathrm{CCl}_{4}$ oral administration, the blood was taken though the mice's eye socket. Then, serum was immediately collected after centrifugation of clotted blood at $3000 \mathrm{rpm}$ at $4{ }^{\circ} \mathrm{C}$ for $15 \mathrm{~min}$. Liver samples were collected immediately from the sacrificed mice and a portion of the left liver lobe was collected and fixed in $10 \%$ formalin for $24 \mathrm{~h}$. The rest of liver was stored at $-80^{\circ} \mathrm{C}$ for subsequent measurement. 
The activities of serum AST and ALT were measured with commercially available test kits. In brief, ALT and AST activities were analyzed by the dinitrophenyl-hydrazine method [35]. The thawed liver sample was homogenized in $0.1 \mathrm{~g} / \mathrm{mL}$ of ice-cold phosphate buffer ( $\mathrm{pH}$ 7.4). The protein content in liver samples was detected by a BCA protein assay kit. The GSH-Px, T-SOD, the levels of MDA and protein content were assayed using commercially available test kits (as indicated in the supplementary material). The liver preserved in 10\% formalin solution was then embedded in paraffin for subsequent slicing and staining with hematoxylin and eosin (H\&E).

\subsection{Data Analysis}

All the data are expressed as the mean \pm standard deviation (SD). The statistical significance of differences between treatment groups in the pharmacokinetic study, tissue distribution and hepatoprotective effect was assessed using an unpaired student's $t$-test. A $p$-value of less than 0.05 represents statistically significant differences.

\section{Results and discussion}

\subsection{Morphology, Particle Size and Zeta Potential}

mPEG2k-LA was synthesized by esterifying mPEG2k with linoleic acid (Figure S8). The critical aggregation concentration (CAC) of $\mathrm{mPEG}_{2 \mathrm{k}}-\mathrm{LA}$ micelle was $0.1372 \mathrm{mg} / \mathrm{mL}$ (Figure S9). The measurement of mean diameter, ZP, EE and DL were among the factors to be considered in the optimal formulation. According to the characterization results of SMs in Table 1, it was shown that the $\mathrm{d}$ and DL of micelles were increased by the percentage of 6-shogaol and $\mathrm{mPEG}_{2 \mathrm{k}}-\mathrm{LA}$. On the contrary, the $\mathrm{ZP}$ and EE decreased as the percentage of $\mathrm{mPEG}_{2 \mathrm{k}}$-LA and 6-shogaol increased. The increase of particle size was from $27.5 \mathrm{~nm}$ at weight rate of $0.05 / 1 \mathrm{~nm}$ to $76.8 \mathrm{~nm}$ at the weight rate of $0.1 / 1$. The remarkable increase of particle size was due to the increase in 6-shogaol encapsulation in the micellar hydrophobic interior [36]. However, the particle size increased slightly from $76.8 \mathrm{~nm}$ at the weight rate of $0.1 / 1$ to $93.51 \mathrm{~nm}$ at $0.2 / 1$. This phenomenon could be potentially due to the absence of 6-shogaol in the micellar core vis à vis its absorbance on the surface of micelles causing ZP to remarkable decrease as the percentage of $\mathrm{mPEG}_{2 \mathrm{k}}-\mathrm{LA}$ and 6-shogaol increased ( $\mathrm{ZP}$ from -3.61 at the rate of $0.1 / 1$ to -11.77 at the rate of $0.2 / 1$ ). Therefore, the rate of $0.1 / 1$ was selected as the optimal formulation ratio of 6-shogaol and $\mathrm{mPEG}_{2 \mathrm{k}}$-LA with appropriate EE (81.6\%), DL (7.3\%) and PDI (0.088). The morphology study using the TEM showed that the SMs were spherical in shape (Figure $1 \mathrm{~A}$ and Figure S10) with particle sizes near to $90 \mathrm{~nm}$, which is close to the results detected via DLS (Figure 1B). Interestingly, it was found that the SMs had a clear core-shell structure which was also consistent with the characterization variation of different percentage of $\mathrm{mPEG}_{2 \mathrm{k}}-\mathrm{LA}$ and 6-shogaol.

A

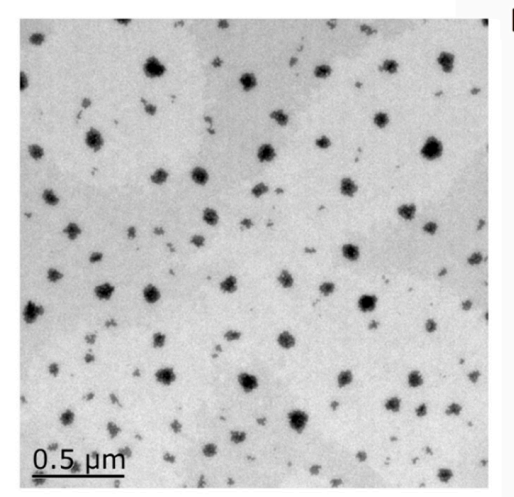

B

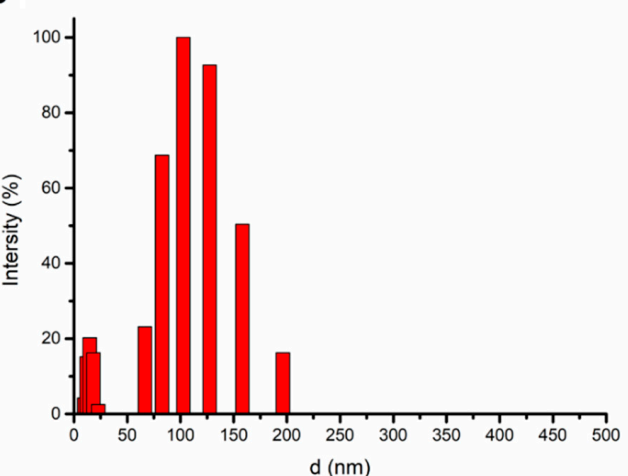

Figure 1. Transmission electron microscope (A) and the dynamic light scattering (B) results of 6-shogaol loaded micelles (SMs). [10/1, the mass of methoxypolyethylene glycol (MW = 2000)-linoleate acid conjugate $\left(\mathrm{mPEG}_{2 \mathrm{k}}\right.$-LA)/6-shogaol]. 
Table 1. Physicochemical characterization of the micelles made of mixtures of 6-shogaol and methoxypolyethylene glycol (MW=2000)-linoleate acid conjugate ( $\left.\mathrm{mPEG}_{2 \mathrm{k}}-\mathrm{LA}\right)$ (mean values \pm standard deviation, $\mathrm{n}=3$ ): measurement of mean diameter $(\mathrm{D})$, Zeta potential $(\mathrm{ZP})$ and polydispersity index (PDI), entrapment efficiency (EE) and drug loading (DL).

\begin{tabular}{ccccccc}
\hline Mass Ratio & Ratio & ZP $(\mathbf{m V})$ & $\mathbf{D}(\mathbf{n m})$ & PDI & EE\% & DL\% \\
\hline & $20: 1$ & -2.05 & 27.54 & 0.105 & 98.4 & 4.7 \\
mPEG $_{2 \mathrm{k}}$-LA/6-shogaol & $10: 1$ & -3.61 & 76.8 & 0.088 & 81.6 & 7.3 \\
& $5: 1$ & -11.77 & 93.51 & 0.120 & 68.9 & 12.4 \\
& $2: 1$ & -12.16 & 104.17 & 0.150 & 35.4 & 15.0 \\
& $1: 1$ & -17.95 & 112.29 & 0.189 & 22.9 & 17.5 \\
\hline
\end{tabular}

\subsection{Solubility Test}

The solubility test results showed that the solubility of 6-shogaol in water, $\mathrm{pH} 1.2$ and 7.4 PBS was $21.39 \pm 2.53 \mu \mathrm{g} / \mathrm{mL}, 26.42 \pm 1.84 \mu \mathrm{g} / \mathrm{mL}$ and $24.76 \pm 3.01 \mu \mathrm{g} / \mathrm{mL}$, respectively. However, the SMs solution prepared at the concentration of $12 \mathrm{mg} / \mathrm{mL}$ was a transparent liquid. This indicated that SMs could markedly improve the solubility of 6-shogaol.

\subsection{In Vitro Release of SMs}

In present study, $\mathrm{pH} 1.2$ and 7.4 phosphate buffer solutions (PBS) were applied to simulate the environment of gastric juice and intestinal fluid, respectively. The in vitro release behavior of 6-shogaol from micelles was evaluated in PBS ( $\mathrm{pH} 1.2$ and 7.4) under sink conditions at $37^{\circ} \mathrm{C}$ for $24 \mathrm{~h}$. The cumulative release result of 6-shogaol from micelles is shown in Figure 2. It was observed that free 6-shogaol in DMSO could release $87 \%$ in artificial intestinal juice during $4 \mathrm{~h}$. It was indicated that 6-shogaol could completely diffuse across the dialysis bag. Over the entire period of the study, a sustained 6-shogaol release totaling approximately $81 \%$ was accumulated from the micelles in the intestinal fluid within $24 \mathrm{~h}$. Notably, the cumulative release rate of 6-shogaol from the micelles in the intestinal juice was higher than that in the artificial gastric juice. According to the results (Figure S7), the peak of $\mathrm{mPEG}_{2 \mathrm{k}}-\mathrm{LA}$ after incubation in $\mathrm{PBS}(\mathrm{pH}=7.4)$ at $37^{\circ} \mathrm{C}$ for $24 \mathrm{~h}$ was longer compared with before incubation. It was observed that the $\mathrm{mPEG}_{2 \mathrm{k}}$-LA begun to degrade to smaller molecular compounds within $24 \mathrm{~h}$, which could potentially serve as the underlying mechanism for the release of 6-shogaol from the micelles. These results indicate that 6-shogaol encapsulated in micelles may be slowly released along the degradation of $\mathrm{mPEG}_{2 \mathrm{k}}-\mathrm{LA}$ which was more easily hydrolyzed in artificial intestinal juice.

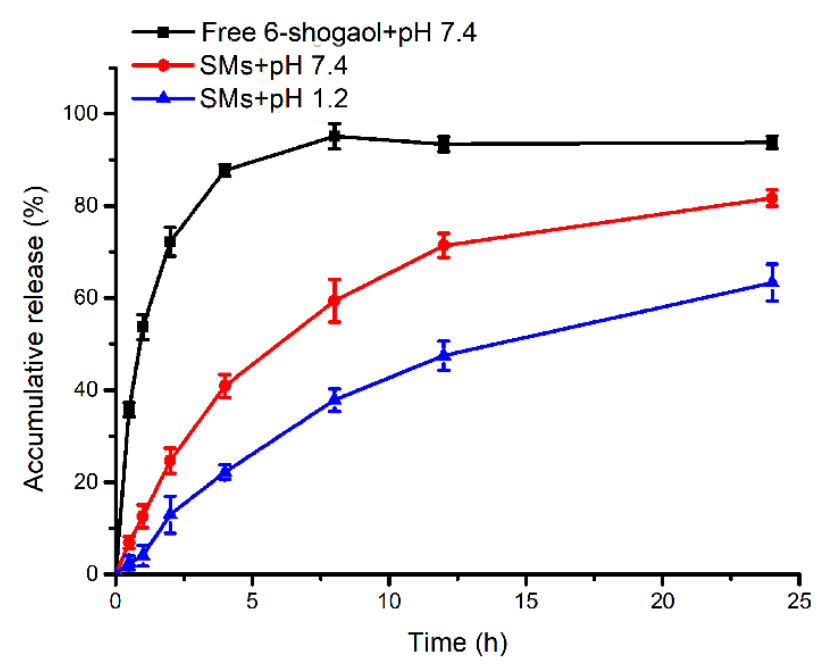

Figure 2. In vitro release of free 6-shogaol and SMs in different medium for $24 \mathrm{~h}$. 


\subsection{In Vitro Anti-Tumor Activity}

The in vitro inhibitory effects of free 6-shogaol and SMs on HepG-2 cells were determined via MTT assay using $\mathrm{mPEG}_{2 \mathrm{k}}$-LA as controls. As show in Figure 3, it was revealed that the proliferation of HepG-2 cells could be inhibited by both free 6-shogaol and SMs in a dose-dependent manner. Astonishingly, the cell viability of SMs on HepG2 was significantly lower than that of free 6-shogaol at low concentration range $(5-50 \mu \mathrm{g} / \mathrm{mL})$. The micelles carrier material $\mathrm{mPEG}_{2 \mathrm{k}}-\mathrm{LA}$ showed almost no cytotoxicity on HepG2 cells at high concentration $(1 \mathrm{mg} / \mathrm{mL})$. Therefore, the reason for the increased cytotoxicity is attributed to its entrapment in micelles with enhanced solubility, rather than the cytotoxicity of the carrier- $\mathrm{mPEG}_{2 \mathrm{k}}-\mathrm{LA}$ [37]. The enhanced in vitro cancer growth inhibition of SMs was possibly due to the different cellular uptake mechanism of the micelles with free 6-shogaol [38]. The encapsulated drug in micelles entered the cell via the carrier-mediated endocytosis due to its extra small size. However, the cellular uptake of free 6-shogaol could be through passive diffusion [39]. Besides, $\mathrm{mPEG}_{2 \mathrm{k}}-\mathrm{LA}$, like some carrier materials including PLGA and TPGS, may have the inhibition ability of active efflux transporters such as p-glycoprotein $[27,40]$. The $\mathrm{mPEG}_{2 \mathrm{k}}$-LA exhibited cytotoxicity at $2 \mathrm{mg} / \mathrm{mL}$ probably owing to the activity of linoleic acid [41].

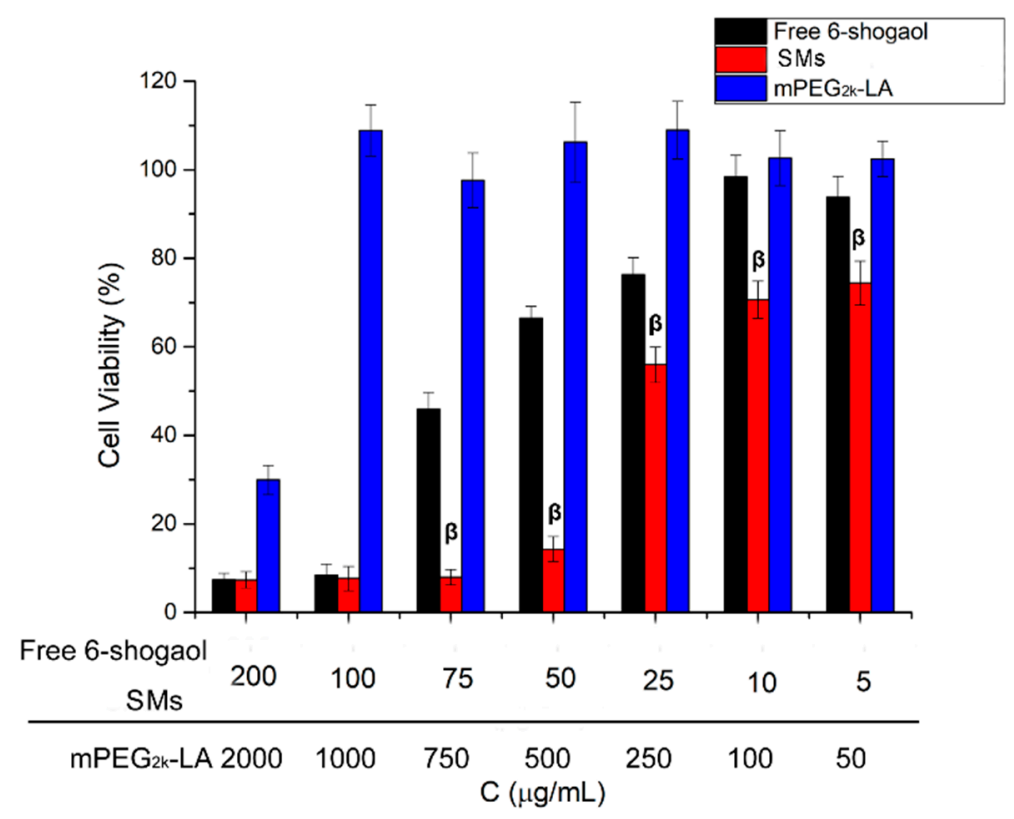

Figure 3. Cell viability of different samples $\left(\mathrm{mPEG}_{2 \mathrm{k}}-\mathrm{LA}\right.$, free 6-shogaol and SMs) with varied concentrations for $72 \mathrm{~h}$ using a 3-(4,5-dimethyl-2-thiazolyl)-2,5-diphenyl-2-H-tetrazolium bromide (MTT) bioassay against HepG2 cells. ${ }^{\beta} P<0.01$ SMs versus free 6-shogaol.

The anti-tumor mechanism of 6-shogaol was found through the induction of cell cycle arrest and apoptosis in human hepatoma cells. 6-shogaol has also been reported to induce apoptosis in human hepatocellular carcinoma cells in relation to caspase activation and endoplasmic reticulum (ER) stress signaling via PERK/eIF2a pathway [42]. Meanwhile, 6-shogaol was established to reduce constitutive and interleukin (IL)-6-induced STAT3 activation while inhibiting both constitutive and TNF-a-induced NF-kB activity to induce the apoptosis of human (LNCaP, DU145, and PC3) and mouse(HMVP2) prostate cancer cells [43].

\subsection{Oral Pharmacokinetic Study of Micelles}

As shown in the plasma concentration-time curves, the plasma 6-shogaol concentration of SMs was greater than that of free 6-shogaol after a $0.75 \mathrm{~h}$ time point (Figure 4 ). The profiles and absorption of SMs also showed significant increases in the parameters $\left(A_{U C} C_{0-12} h, t_{1 / 2}, M R T, T_{\max }, C_{\max }\right)$ (Table 2). Specifically, the SMs took a 2.78-fold longer time to reach the maximum plasma concentration compared 
with the free 6-shogaol. The $\mathrm{C}_{\max }$ of the encapsulated 6-shogaol was also approximately 1.18-fold higher than the free drug suspension, while the $\mathrm{AUC}_{0-12 \mathrm{~h}}$ also showed an approximate 3.2-fold increase. Importantly, $\mathrm{t}_{1 / 2}$ and MRT were significantly prolonged after forming the micelles due to the slower release rate compared to the free 6-shogaol suspension. The enhancement of absorption and bioavailability could be ascribed to the increase in solubility and small particle size [44]. Collectively, these findings suggest that SMs could significantly improve the oral bioavailability and absorption with the potential to further enhance tissue distribution and in vivo bioactivity.

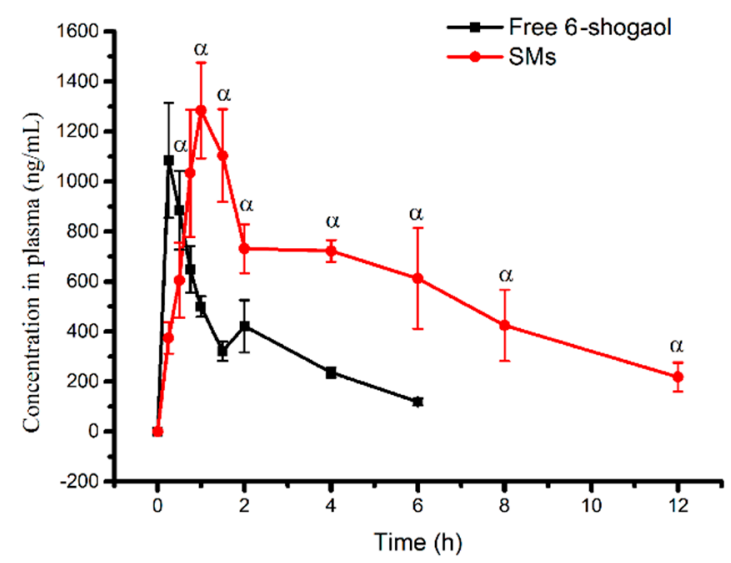

Figure 4. Mean plasma concentration-time profiles of 6-shogaol after oral administration of a single $100 \mathrm{mg} / \mathrm{kg}$ dose each of SMs and free 6-shogaol to rats. Results are presented as the Mean \pm SD $(\mathrm{n}=5)$. ${ }^{\alpha} P<0.05$, SMs versus free 6-shogaol.

Table 2. Pharmacokinetic parameters of free 6-shogaol and 6-shogaol loaded micelles (SMs) after oral administration in rats (mean $\pm \mathrm{SD}$ ).

\begin{tabular}{ccc}
\hline Parameters & Free Drug & SMs \\
\hline $\mathrm{C}_{\max }\left(\mathrm{ng} \cdot \mathrm{mL}^{-1}\right)$ & $1085.3 \pm 230.1$ & $1284.3 \pm 191^{\alpha}$ \\
$\mathrm{T}_{\max }(\mathrm{h})$ & $0.33 \pm 0.14$ & $0.92 \pm 0.14^{\beta}$ \\
$\mathrm{t}_{1 / 2}(\mathrm{~h})$ & $2.05 \pm 0.09$ & $4.65 \pm 0.39^{\beta}$ \\
$\mathrm{MRT}(\mathrm{h})$ & $2.06 \pm 0.05$ & $7.48 \pm 2.33^{\beta}$ \\
$\mathrm{AUC}_{0-4} \mathrm{~h}\left(\mathrm{~h} \cdot \mathrm{ng} \cdot \mathrm{mL}^{-1}\right)$ & $2123.2 \pm 193.6$ & $6834.1 \pm 826.1^{\beta}$ \\
\hline$\alpha{ }^{\alpha}<0.05$, compared with free 6-shogaol. ${ }^{\beta} P<0.01$, compared with free6-shogaol.
\end{tabular}

\subsection{Tissue Distribution of SMs}

The tissue distribution results of free 6-shogaol and SMs after oral administration at $0.5 \mathrm{~h}, 1 \mathrm{~h}$ and $2 \mathrm{~h}$ are shown in Figure 5, respectively. It was observed that free 6-shogaol accumulated significantly in the stomach, small intestine and liver at $0.5 \mathrm{~h}$ and $1 \mathrm{~h}$ due to the liver first pass effect. However, the liver distribution of free 6-shogaol at $2 \mathrm{~h}$ declined sharply and was lower than other tissues such as heart, spleen, lung, kidney and brain. Notably, the 6-shogaol was mostly distributed in the kidney with the exception of the stomach at $2 \mathrm{~h}$. This indicated that free 6-shogaol would be rapidly catabolized in the liver and excreted in the kidney. The tissue distribution results of free 6-shogaol was consistent with other reports [45]. Compared to free 6-shogaol, the tissue distribution of SMs in the liver, lung and stomach was significantly enhanced due to the ability of the micelle to improve the solubility and oral absorption of the lipophilic drug. Furthermore, the relative liver delivery index (DDI) of SMs at $2 \mathrm{~h}$ was significantly higher than that of the free 6-shogaol (relative to blood, $1.45 \mathrm{vs} .0 .69, \mathrm{c} / \mathrm{c}$ ). In contrast, the relative kidney distribution of SMs at $2 \mathrm{~h}$ was significantly lower than that of free 6-shogaol (relative to blood, 0.73 vs 1.53, c/c). More significantly, the distribution in brain of 6-shogaol was enhanced after its encapsulating in the SMs. This is because smaller micelles (50 nm-100 nm) could enter the lymphatic system in the intestine and delivery 6-shogaol into the brain and liver [22]. 
These results demonstrated that SMs could improve the accumulation of 6-shogaol in tissue especially the brain and liver tissue.

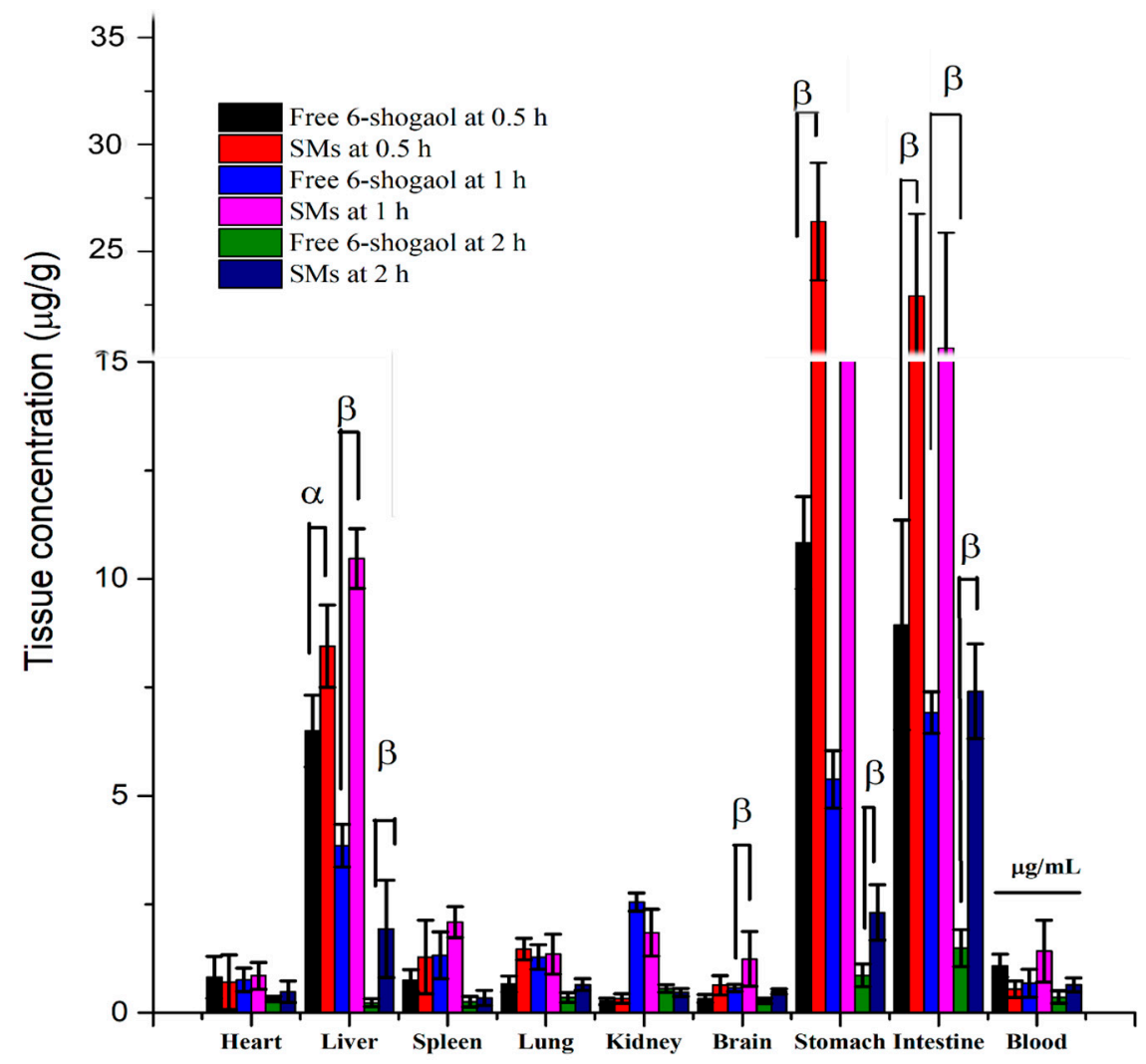

Figure 5. Concentration of 6-shogaol in the heart, liver, spleen, lung, kidney, stomach, small intestine brain and blood following oral administration of a single $100 \mathrm{mg} / \mathrm{kg}$ dose of free 6-shogaol and SMs to mice at $0.5 \mathrm{~h}, 1 \mathrm{~h}$ and $2 \mathrm{~h}$ point. Results are presented as the Mean $\pm \mathrm{SD}(\mathrm{n}=5) .{ }^{\alpha} P<0.05 \mathrm{SM}$ s versus free 6-shogaol. ${ }^{\beta} P<0.01$ SMs versus free 6-shogaol.

\subsection{Hepatoprotective Effect in Vivo}

The results of the hepatoprotective effect of 6-shogaol and SMs on the serum ALT and AST activities are shown in Figure 6A. In the $\mathrm{CCl}_{4}$ intoxicated group, serum ALT and AST values were $318.5 \mathrm{U} / \mathrm{L}$ and 161.2 U/L, respectively, whereas the values of normal group were only $25.49 \mathrm{U} / \mathrm{L}$ and $15.46 \mathrm{U} / \mathrm{L}$, respectively. The significant increase in the activities of serum ALT and AST indicated that the $\mathrm{CCl}_{4}$ induced liver injury model was successfully established. Oral administration of positive drug sylimarin, 6-shogaol and SMs for one week significantly reduced the activities of serum ALT by $28.4 \%$, $36.0 \%$ and $41.78 \%$, and as well as the activities of serum AST by $33.3 \%, 28.0 \%$ and $45.9 \%$, respectively, as compared to the model group $(P<0.05)$. More importantly, the activities of serum ALT and AST in the SMs group was significantly reduced compared to the 6-shogaol group $(P<0.05)$.

The activities of GSH-Px, T-SOD and MDA in the liver were also evaluated. Significantly lowered activities of GSH-Px and T-SOD, coupled with increased MDA content were observed in $\mathrm{CCl}_{4}$ model group mice liver as compared with the control group (Figure 6B-D). The positive drug sylimarin, 6-shogaol and SMs also showed a significant increase in the activities of GSH-Px and T-SOD as well as a decrease in the content of MDA in the liver compared to the model group $(P<0.05)$. Notably, the SMs showed higher activities of GSH-Px and T-SOD as well as a lower content of MDA in the liver than the 6-shogaol group, even more than the positive cohort $(P<0.05)$. 

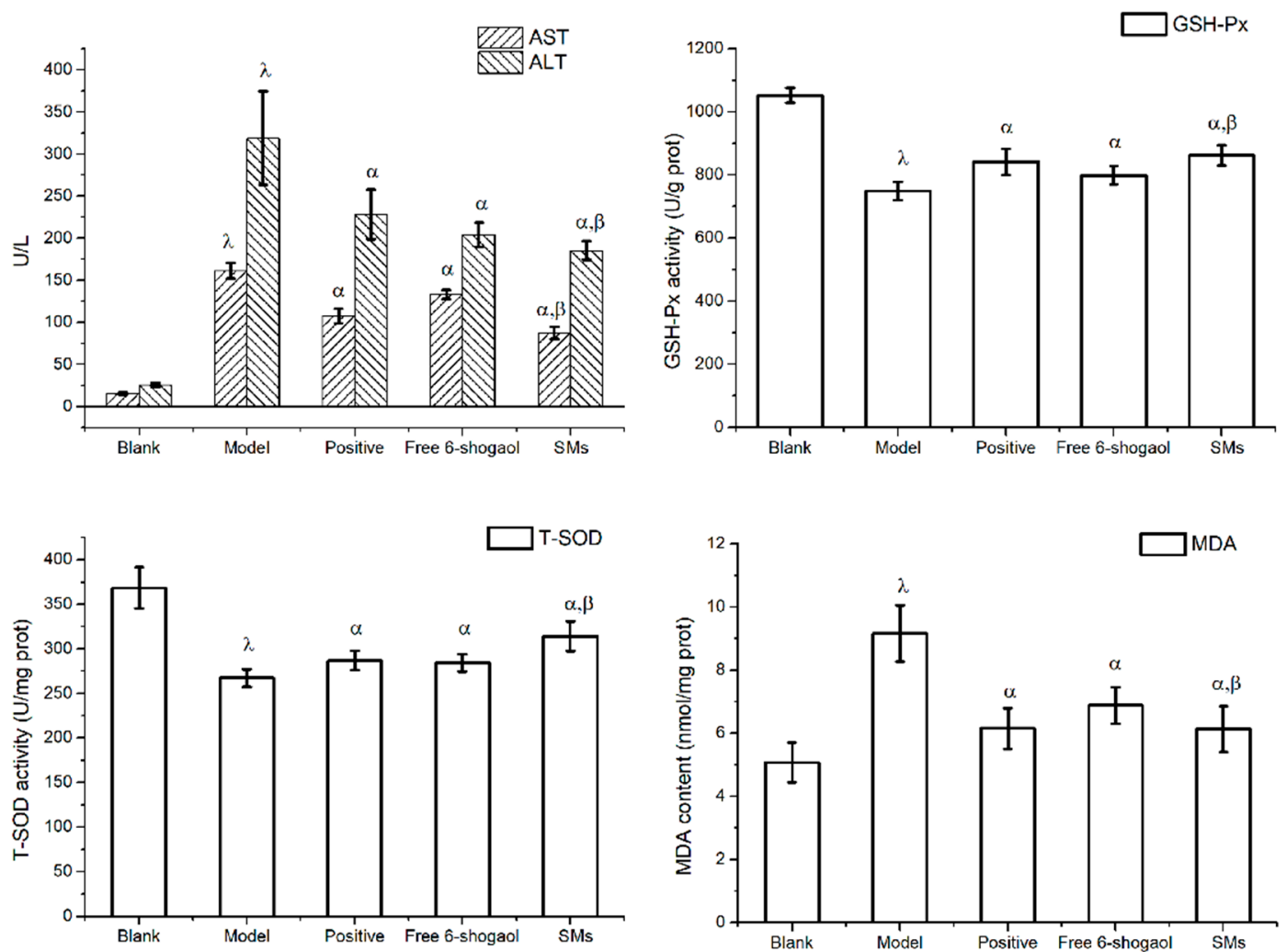

Figure 6. Effects of free 6-shogaol and SMs on serum ALT, AST activity (A) and liver GSH-Px (B), T-SOD (C) and MDA (D) activity. Animals were given orally: castor oil (model group); silymarin (positive group, $100 \mathrm{mg} / \mathrm{kg}$ ); free 6-shogaol (100 mg/kg); or SMs (100 mg/kg) once daily for one week prior to the administration of $\mathrm{CCl}_{4}(0.2 \%$, i.p). Except blank group, which accepted the administration of castor oil. ${ }^{\alpha} P<0.05$ versus model group. ${ }^{\beta} P<0.01$ versus free 6 -shogaol. ${ }^{\lambda} P<0.05$ model group versus blank group.

The hepatoprotective effects of 6-shogaol and SMs were further confirmed using conventional histological assessment (Figure 7). The histology of the liver sections of the control group showed normal hepatic cells with a well-preserved cytoplasm and a prominent nucleus and nucleolus (Figure 7A). The stained sections of $\mathrm{CCl}_{4}$ model group revealed extensive liver injuries characterized by obvious soma shrinking, dissociation of the hepatic cord, large area hepatic necrosis, inflammatory cell infiltration and ballooning degeneration (Figure $7 \mathrm{~B}$ ). $\mathrm{CCl}_{4}$-intoxicated mice pretreated with 6-shogaol and positive group showed moderate hypertrophy of hepatocytes with a relatively intact central vein, diminished hepatic necrosis and reduced inflammatory cell (Figure $7 \mathrm{C}$ ). However, $\mathrm{CCl}_{4}$-intoxicated mice pretreated with SMs only exhibited individual cell ballooning degeneration compared to $\mathrm{CCl}_{4}$ model group. These results indicated that 6-shogaol has certain hepatoprotective effects against the $\mathrm{CCl}_{4}$-induced liver injury through restoration and maintenance of the activities of T-SOD and GSH-Px in $\mathrm{CCl}_{4}$-damaged liver. The underlying mechanism of hepatoprotective effects of 6-shogaol might be attributed to anti-inflammatory and antioxidative activities of its phenolic structure [46,47]. More significantly, SMs might improve the hepatoprotective effects of 6-shogaol due to enhanced oral bioavailability coupled with liver accumulation. 

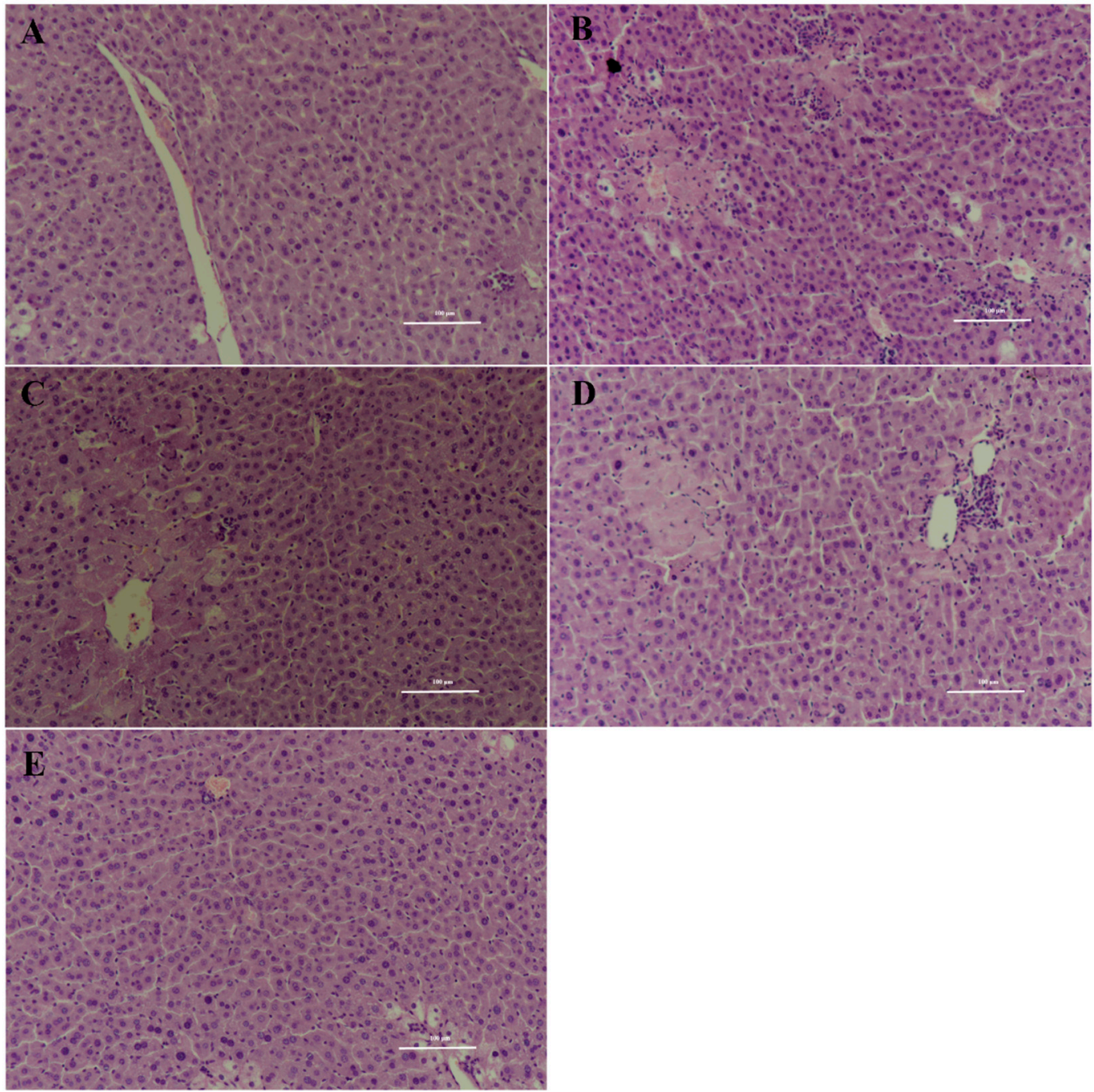

Figure 7. Effects of free 6-shogaol and SMs on hepatic morphological analysis $(\times 200 \mathrm{H \& E})$ : Blank group (A), $\mathrm{CCl}_{4}$-model group (B), Positive drug $(100 \mathrm{mg} / \mathrm{kg})$ and $\mathrm{CCl}_{4}$ group (C), Free 6-shogaol $(100 \mathrm{mg} / \mathrm{kg})$ and $\mathrm{CCl}_{4}$ group (D), SMs $(100 \mathrm{mg} / \mathrm{kg})$ and $\mathrm{CCl}_{4}$ group (E). The scale bar: $100 \mu \mathrm{m}$.

\section{Conclusions}

Novel $\mathrm{mPEG}_{2 \mathrm{k}}$-LA micelles were successfully developed for oral delivery of the hydrophobic compound 6-shogaol. A high drug encapsulation of $80 \%$ was achieved under drug loading capacity of $7 \%$, which greatly enhanced the 6-shogaol delivery efficiency versus general oral delivery systems. The developed micelles (SMs) showed a slower release rate than the free 6-shogaol. Moreover, SMs significantly improved the anti-cancer activity of 6-shogaol in vitro. In addition, SMs showed enhanced oral bioavailability and liver distribution compared to free 6-shogaol. The in vivo liver protection study also demonstrated that SMs markedly reduced the activities of serum AST, ALT and liver MDA levels, while remarkably increased the antioxidant activities (GSH-Px, T-SOD). Therefore, the novel micelle is expected to serve as a promising carrier for 6-shogaol to enhance its cancer treatment and hepatoprotection. 
Supplementary Materials: The following are available online at http:/ /www.mdpi.com/1999-4923/11/3/107/s1, Figure S1: The ESI-MS of 6-shogaol, Figure S2: The ${ }^{1} \mathrm{H}-\mathrm{NMR}$ of 6-shogaol (CDCl3, ppm), Figure S3: The chromatogram of purified 6-shogaol and the structure of 6-shogaol (insert), Figure S4: HPLC chromatograms of blank micelles (A), 6-shogaol (B) and sample of 6-shogaol-loaded micelles (C), Figure S5: Chromatograms of 6-shogaol and internal standard in rat plasma, Figure S6: Chromatograms of 6-shogaol and internal standard in mouse heart, liver, spleen, lung, kidney, brain, stomach, intestine homogenate and plasma. Figure S7: The gel chromatogram of $\mathrm{mPEG}_{2 \mathrm{k}}-\mathrm{LA}$ and $\mathrm{mPEG}_{2 \mathrm{k}}-\mathrm{LA}$ after incubation at PBS $=7.4$ for $24 \mathrm{~h}$, Figure S8: Synthesis route of mPEG2k-LA, Figure S9: The critical aggregation concentration (CAC) of $\mathrm{mPEG}_{2 \mathrm{k}}$-LA micelles was measured by Fluorescence probes (pyrene), Figure S10: The TEM micrograph of 6-shogaol loaded micelles (6-shgoaol/ $\mathrm{mPEG}_{2 \mathrm{k}}$-LA = 1/10). Table S1: Linear ranges, standard curves and regression coefficients of 6-shogaol in different biosamples. Table S2: The precision (intra-day and inter-day precision), accuracy (relative recovery) and extract recovery of analysis method in vivo.

Author Contributions: For research articles with several authors, a short paragraph specifying their individual contributions must be provided. Conceptualization, X.X. and J.Y.; methodology, H.Z., Q.W. (Qilong Wang) and C.S.; analysis, H.Z., Q.Y., and Q.W. (Qiuyu Wei); data curation, H.Z., Y.Z. and J.C.; writing-original draft preparation, H.Z., W.D. and M.A.-F.; writing-review and editing, X.X. and J.Y.; supervision, X.X. and J.Y.

Funding: This work was kindly supported by the National Natural Science Foundation of China (30973677, 81373371), China Postdoctoral Science Foundation (2018M630533), Program for Scientific research innovation team in Colleges and Universities of Jiangsu Province as well as A Project Funded by the Priority Academic Program Development of Jiangsu Higher Education Institutions.

Conflicts of Interest: The authors declare that they have no conflicts of interest.

\section{Abbreviations}

SMs, 6-shogaol loaded in micelles;

DL, Drug loading;

$\mathrm{ZP}$, zeta potential;

$\mathrm{mPEG}_{2 \mathrm{~K}}-\mathrm{LA}$, methoxypolyethylene glycol $(\mathrm{MW}=2000)$-linoleate acid conjugate linoleate acid conjugate;

FBS, fetal bovine serum;

PBS, phosphate buffer saline;

HPLC, high performance liquid chromatography;

PDI, polydispersity index;

TEM, transmission electron microscopy;

ESI-MS, Electrospray Ionization Mass Spectrometry;

NMR, nuclear magnetic resonance spectroscopy;

DMSO, dimethylsulfoxide;

rpm, revolutions per minute;

MRT, mean residence time

\section{References}

1. Li, Z.; Wang, Y.; Gao, M.; Cui, W.; Zeng, M.; Cheng, Y.; Li, J. Nine New Gingerols from the Rhizoma of Zingiber officinale and Their Cytotoxic Activities. Molecules 2018, 23, 315. [CrossRef] [PubMed]

2. Dedov, V.N.; Tran, V.H.; Duke, C.C.; Connor, M.; Christie, M.D.J.; Mandadi, S.; Roufogalis, B.D. Gingerols: A novel class of vanilloid receptor (VR1) agonists. Br. J. Pharmacol. 2002, 137, 793-798. [CrossRef] [PubMed]

3. Jiang, Y.; Liao, Q.; Zou, Y. Transcriptome analysis reveals the genetic basis underlying the biosynthesis of volatile oil, gingerols, and diarylheptanoids in ginger (Zingiber officinale Rosc.). Bot. Stud. 2017, 58, 41-52. [CrossRef] [PubMed]

4. Yamahara, J.; Matsuda, H.; Yamaguchi, S.; Shimoda, H.; Murakami, N.; Yoshikawa, M. Pharmacological Study on Ginger Processing. I.: Antiallergic Activity and Cardiotonic Action of Gingerols and Shogaols. Nat. Med. 1995, 49, 76-83.

5. Jung, M.Y.; Min, K.L.; Park, H.J.; Oh, E.B.; Shin, J.Y.; Ji, S.P.; Su, Y.J.; Oh, J.H.; Choi, D.S. Heat-induced conversion of gingerols to shogaols in ginger as affected by heat type (dry or moist heat), sample type (fresh or dried), temperature and time. Food Sci. Biotechnol. 2018, 27, 687-693. [CrossRef] [PubMed]

6. Sang, S.; Hong, J.; Wu, H.; Liu, J.; Yang, C.S.; Pan, M.H.; Badmaev, V.; Ho, C.T. Increased growth inhibitory effects on human cancer cells and anti-inflammatory potency of shogaols from Zingiber officinale relative to gingerols. J. Agric. Food Chem. 2009, 57, 10645-10650. [CrossRef] [PubMed] 
7. Sakulnarmrat, K.; Srzednicki, G.; Konczak, I. Antioxidant, enzyme inhibitory and antiproliferative activity of polyphenolic-rich fraction of commercial dry ginger powder. Int. J. Food Sci. Technol. 2015, 50, 2229-2235. [CrossRef]

8. Wu, J.J.; Omar, H.A.; Lee, Y.R.; Teng, Y.N.; Chen, P.S.; Chen, Y.C.; Huang, H.S.; Lee, K.H.; Hung, J.H. 6-Shogaol induces cell cycle arrest and apoptosis in human hepatoma cells through pleiotropic mechanisms. Eur. J. Pharmacol. 2015, 762, 449-458. [CrossRef] [PubMed]

9. Li, T.Y.; Chiang, B.H. 6-shogaol induces autophagic cell death then triggered apoptosis in colorectal adenocarcinoma HT-29 cells. Biomed. Pharmacother. 2017, 93, 208-217. [CrossRef] [PubMed]

10. Hung, J.Y.; Hsu, Y.L.; Li, C.T.; Ko, Y.C.; Ni, W.C.; Huang, M.S.; Kuo, P.L. 6-Shogaol, an active constituent of dietary ginger, induces autophagy by inhibiting the AKT/mTOR pathway in human non-small cell lung cancer A549 cells. J. Agric. Food Chem. 2009, 57, 9809. [CrossRef] [PubMed]

11. Pan, M.H.; Hsieh, M.C.; Hsu, P.C.; Ho, S.Y.; Lai, C.S.; Wu, H.; Sang, S.; Ho, C.T. 6-Shogaol suppressed lipopolysaccharide-induced up-expression of iNOS and COX-2 in murine macrophages. Mol. Nutr. Food Res. 2008, 52, 1467-1477. [CrossRef] [PubMed]

12. Sabina, E.P.; Rasool, M.; Mathew, L.; Ezilrani, P.; Indu, H. 6-Shogaol inhibits monosodium urate crystal-induced inflammation-An in vivo and in vitro study. Food Chem. Toxicol. 2010, 48, 229-235. [CrossRef] [PubMed]

13. Prasad, S.; Tyagi, A.K. Ginger and Its Constituents: Role in Prevention and Treatment of Gastrointestinal Cancer. Gastroent. Res. Pract. 2015, 2015, 142979-142989. [CrossRef] [PubMed]

14. Cheng, Y.; Oh, J.; Oh, I.G.; Park, C.; Jin, H.C. [6]-Shogaol inhibits melanogenesis in B16 mouse melanoma cells through activation of the ERK pathway. Acta Pharmacol. Sin. 2013, 34, 289-294.

15. Wu, C.H.; Hong, B.H.; Ho, C.T.; Yen, G.C. Targeting cancer stem cells in breast cancer: Potential anticancer properties of 6-shogaol and pterostilbene. J. Agric. Food Chem. 2015, 63, 2432-2441. [CrossRef] [PubMed]

16. Kim, M.O.; Lee, M.H.; Oi, N.; Kim, S.H.; Bae, K.B.; Huang, Z.; Dong, J.K.; Reddy, K.; Lee, S.Y.; Si, J.P. [6]-Shogaol inhibits growth and induces apoptosis of non-small cell lung cancer cells by directly regulating Akt1/2. Carcinogenesis 2014, 35, 683-691. [CrossRef] [PubMed]

17. Kotowski, U.; Kadletz, L.; Schneider, S.; Foki, E.; Schmid, R.; Seemann, R.; Thurnher, D.; Heiduschka, G. 6-shogaol induces apoptosis and enhances radiosensitivity in head and neck squamous cell carcinoma cell lines. Phytol. Res. 2018, 32, 340-347. [CrossRef] [PubMed]

18. Wu, H.; Hsieh, M.C.; Lo, C.Y.; Liu, C.B.; Sang, S.; Ho, C.T.; Pan, M.H. 6-Shogaol is more effective than 6-gingerol and curcumin in inhibiting 12-O-tetradecanoylphorbol 13-acetate-induced tumor promotion in mice. Mol. Nutr. Food Res. 2010, 54, 1296-1306. [CrossRef] [PubMed]

19. Chen, H.; Lv, L.; Soroka, D.; Warin, R.F.; Parks, T.A.; Hu, Y.; Zhu, Y.; Chen, X.; Sang, S. Metabolism of [6]-shogaol in mice and in cancer cells. Drug Metab. Dispos. 2012, 40, 742-753. [CrossRef] [PubMed]

20. Asami, A.; Shimada, T.; Mizuhara, Y.; Asano, T.; Takeda, S.; Aburada, T.; Miyamoto, K.; Aburada, M. Pharmacokinetics of [6]-shogaol, a pungent ingredient of Zingiber officinale Roscoe (Part I). J. Nat. Med. 2010, 64, 281-287. [CrossRef] [PubMed]

21. Zhu, S.Y.; Dong, Y.; Tu, J.; Zhou, Y.; Zhou, X.H.; Xu, B. Silybum marianum oil attenuates oxidative stress and ameliorates mitochondrial dysfunction in mice treated with D-galactose. Pharmacogn. Mag. 2014, 10, S92-S99. [PubMed]

22. Yi, C.; Zhong, H.; Tong, S.; Cao, X.; Firempong, C.K.; Liu, H.; Fu, M.; Yang, Y.; Feng, Y.; Zhang, H. Enhanced oral bioavailability of a sterol-loaded microemulsion formulation of Flammulina velutipes, a potential antitumor drug. Int. J. Nanomed. 2012, 7, 5067-5078.

23. Yi, C.; Sun, C.; Tong, S.; Cao, X.; Feng, Y.; Firempong, C.K.; Jiang, X.; Xu, X.; Yu, J. Cytotoxic effect of novel Flammulina velutipes sterols and its oral bioavailability via mixed micellar nanoformulation. Int. J. Pharm. 2013, 448, 44-50. [CrossRef] [PubMed]

24. Hou, J.; Wang, J.; Sun, E.; Yang, L.; Yan, H.M.; Jia, X.B.; Zhang, Z.H. Preparation and evaluation of icariside II-loaded binary mixed micelles using Solutol ${ }^{\circledR}$ HS15 and Pluronic F127 as carriers. Drug Deliv. 2016, 23, 3248-3256. [CrossRef] [PubMed]

25. Hou, J.; Sun, E.; Sun, C.; Wang, J.; Yang, L.; Jia, X.B.; Zhang, Z.H. Improved oral bioavailability and anticancer efficacy on breast cancer of paclitaxel via Novel Soluplus $\left({ }^{\circledR}\right)$-Solutol $\left({ }^{\circledR}\right)$ HS15 binary mixed micelles system. Int. J. Pharm. 2016, 512, 186-193. [CrossRef] [PubMed] 
26. Wang, G.; Wang, J.J.; Chen, X.L.; Li, D.; Fei, L. Quercetin-loaded freeze-dried nanomicelles: Improving absorption and anti-glioma efficiency in vitro and in vivo. J. Control. Release 2016, 235, 276-290. [CrossRef] [PubMed]

27. Zhang, Z.; Tan, S.; Feng, S.S. Vitamin E TPGS as a molecular biomaterial for drug delivery. Biomaterials 2012, 33, 4889-4906. [CrossRef] [PubMed]

28. Gao, A.X.; Liao, L.; Johnson, J.A. Synthesis of Acid-Labile PEG and PEG-Doxorubicin-Conjugate Nanoparticles via Brush-First ROMP. Acs Macro Lett. 2014, 3, 854-857. [CrossRef] [PubMed]

29. Li, Z.; Han, X.; Zhai, Y.; Lian, H.; Zhang, D.; Zhang, W.; Wang, Y.; He, Z.; Liu, Z.; Sun, J. Critical determinant of intestinal permeability and oral bioavailability of pegylated all trans-retinoic acid prodrug-based nanomicelles: Chain length of poly (ethylene glycol) corona. Colloids Surf. B Biointerfaces 2015, 130, 133-140. [CrossRef] [PubMed]

30. Mi, Y.; Liu, Y.; Feng, S.S. Formulation of Docetaxel by folic acid-conjugated d- $\alpha$-tocopheryl polyethylene glycol succinate 2000 (Vitamin E TPGS(2k)) micelles for targeted and synergistic chemotherapy. Biomaterials 2011, 32, 4058-4066. [CrossRef] [PubMed]

31. Varma, M.V.; Panchagnula, R. Enhanced oral paclitaxel absorption with vitamin E-TPGS: Effect on solubility and permeability in vitro, in situ and in vivo. Eur. J. Pharm. Sci. 2005, 25, 445-453. [CrossRef] [PubMed]

32. Zhang, H.; Xu, W.; Omarisiaw, E.; Liu, Y.; Chen, B.; Chen, D.; Yu, J.; Xu, X. Redox-responsive PEGylated self-assembled prodrug-nanoparticles formed by single disulfide bond bridge periplocymarin-vitamin $\mathrm{E}$ conjugate for liver cancer chemotherapy. Drug Deliv. 2017, 24, 1170-1178. [CrossRef] [PubMed]

33. Zhang, P.; Huang, Y.; Liu, H.; Marquez, R.T.; Lu, J.; Zhao, W.; Zhang, X.; Gao, X.; Li, J.; Venkataramanan, R. A PEG-Fmoc conjugate as a nanocarrier for paclitaxel. Biomaterials 2014, 35, 7146-7156. [CrossRef] [PubMed]

34. Zhang, H.Y.; Xu, W.Q.; Zheng, Y.Y.; Omari-Siaw, E.; Zhu, Y.; Cao, X.; Tong, S.S.; Yu, J.N.; Xu, X.M. Octreotide-periplocymarin conjugate prodrug for improving targetability and anti-tumor efficiency: Synthesis, in vitro and in vivo evaluation. Oncotarget 2016, 7, 86326-86338. [CrossRef] [PubMed]

35. Maheshwari, D.T.; Kumar, M.S.Y.; Verma, S.K.; Singh, V.K.; Singh, S.N. Antioxidant and hepatoprotective activities of phenolic rich fraction of Seabuckthorn (Hippophae rhamnoides L.) leaves. Food Chem. Toxicol. 2011, 49, 2422-2428. [CrossRef] [PubMed]

36. Pagels, R.F.; Edelstein, J.; Tang, C.; Prud'Homme, R.K. Controlling and Predicting Nanoparticle Formation by Block Copolymer Directed Rapid Precipitations. Nano Lett. 2018, 18, 1139-1144. [CrossRef] [PubMed]

37. Wang, Q.; Wei, Q.; Yang, Q.; Cao, X.; Li, Q.; Shi, F.; Tong, S.; Feng, C.; Yu, Q.; Yu, J. A novel formulation of [6]-gingerol: Proliposomes with enhanced oral bioavailability and antitumor effect. Int. J. Pharm. 2017, 535, 308-315. [CrossRef] [PubMed]

38. Zhang, H.Y.; Firempong, C.K.; Wang, Y.W.; Xu, W.Q.; Wang, M.M. Ergosterol-loaded poly(lactide-co-glycolide) nanoparticles with enhanced in vitro antitumor activity and oral bioavailability. Acta Pharmacol. Sin. 2016, 37, 834-844. [CrossRef] [PubMed]

39. Hou, J.; Sun, E.; Zhang, Z.H.; Wang, J.; Yang, L.; Cui, L.; Ke, Z.C.; Tan, X.B.; Jia, X.B.; Lv, H. Improved oral absorption and anti-lung cancer activity of paclitaxel-loaded mixed micelles. Drug Deliv. 2017, 24, 261-269. [CrossRef] [PubMed]

40. Xie, X.; Tao, Q.; Zou, Y.; Zhang, F.; Guo, M.; Wang, Y.; Wang, H.; Zhou, Q.; Yu, S. PLGA Nanoparticles Improve the Oral Bioavailability of Curcumin in Rats: Characterizations and Mechanisms. J. Agric. Food Chem. 2011, 59, 9280-9289. [CrossRef] [PubMed]

41. Shultz, T.D.; Chew, B.P.; Seaman, W.R. Differential stimulatory and inhibitory responses of human MCF-7 breast cancer cells to linoleic acid and conjugated linoleic acid in culture. Anticancer Res. 1992, 12, 2143-2145. [PubMed]

42. Pan, M.H.; Hsieh, M.C.; Kuo, J.M.; Lai, C.S.; Wu, H.; Sang, S.; Ho, C.T. 6-Shogaol induces apoptosis in human colorectal carcinoma cells via ROS production, caspase activation, and GADD 153 expression. Mol. Nutr. Food Res. 2010, 52, 527-537. [CrossRef] [PubMed]

43. Saha, A.; Blando, J.; Silver, E.; Beltran, L.; Sessler, J.; Digiovanni, J. 6-Shogaol from dried ginger inhibits growth of prostate cancer cells both in vitro and in vivo through inhibition of STAT3 and NF-KB signaling. Cancer Prev. Res. 2014, 7, 627-638. [CrossRef] [PubMed]

44. Zhu, Y.; Peng, W.; Zhang, J.; Wang, M.; Firempong, C.K.; Feng, C.; Liu, H.; Xu, X.; Yu, J. Enhanced oral bioavailability of capsaicin in mixed polymeric micelles: Preparation, in vitro and in vivo evaluation. J. Funct. Foods 2014, 8, 358-366. [CrossRef] 
45. Nikam, A.R.; Sathiyanarayanan, L.; Mahadik, K.R. Mapping pharmacokinetic and tissue distribution profile of [6]-shogaol from ginger oleoresin. Int. J. Pharm. Pharm. Sci. 2013, 5, 185-189.

46. Li, F.; Nitteranon, V.; Tang, X.; Liang, J.; Zhang, G.; Parkin, K.L.; Hu, Q. In vitro antioxidant and anti-inflammatory activities of 1-dehydro-[6]-gingerdione, 6-shogaol, 6-dehydroshogaol and hexahydrocurcumin. Food Chem. 2012, 135, 332-337. [CrossRef] [PubMed]

47. Zhang, G.; Nitteranon, V.; Chan, L.Y.; Parkin, K.L. Glutathione conjugation attenuates biological activities of 6-dehydroshogaol from ginger. Food Chem. 2013, 140, 1-8. [CrossRef] [PubMed]

(C) 2019 by the authors. Licensee MDPI, Basel, Switzerland. This article is an open access article distributed under the terms and conditions of the Creative Commons Attribution (CC BY) license (http:/ / creativecommons.org/licenses/by/4.0/). 